\title{
Epidermal Growth Factor Outperforms Placebo in the Treatment of Diabetic Foot Ulcer: A Meta-Analysis
}

\author{
Fazal Rahim ${ }^{1}$, Xie Yan ${ }^{2,3}$, Nida Bibi ${ }^{4}$, Shah Nawaz ${ }^{5}$ and Yao Ming $^{1^{*}}$ \\ ${ }^{1}$ Burn and Plastic Surgery Department, General Hospital of Ningxia Medical University, Yinchuan, P.R. China \\ ${ }^{2}$ Tissue Organ Bank \& Tissue Engineering Centre, General Hospital of Ningxia Medical University, Yinchuan, P.R. China \\ ${ }^{3}$ School of Biomedical Sciences, Faculty of Health, Queensland University of Technology, Brisbane, Australia \\ ${ }^{4}$ Department of Zoology, Shaheed Benazir Bhutto University, Sheringal 18000, Pakistan \\ ${ }^{5}$ Department of Infectious Diseases, General Hospital of Ningxia Medical University, Yinchuan, P.R. China
}

*Corresponding author: Prof. Yao Ming, Burn and Plastic Surgery Department, General Hospital of Ningxia Medical University, Yinchuan, P.R. China, Tel: +86-13995015155

\begin{abstract}
A diabetic foot ulcer is a life-threatening ailment caused by the widespread prevalence of diabetes. To overcome the inherent problem, several growth factors, as well as their various combinations, have showed promising effect in aiding diabetic foot ulcer healing. However, contradictory or paradoxical results are often available, debates about this issue have never ceased so far. Therefore, a comprehensive meta-analysis was performed to compare the efficacy and safety of epidermal growth factor and placebo in healing diabetic foot ulcers. The search database includes relevant English literature from Cochrane Library, PubMed, Google Scholar, Elsevier, and EMBASE that was published between 2009 and 2021. The complete healing rate of DFUs was the primary outcome of interest in this study. Eight randomized control trials (RCTs) that involved 620 patients (337 in the EGF group and 283 in the placebo group), were included in this meta-analysis. The results suggest that EGF achieved a higher complete healing rate than placebo after four weeks of treatment (relative risk: $3.04(0.50,18.44)$ and heterogeneity $\left(\mathrm{Chi}^{2}=6.46, \mathrm{df}=2(\mathrm{P}=0.04) \mathrm{I}^{2}=69 \%\right)$; likewise, after eight weeks of treatment, the relative risk and heterogeneity were RR: $2.59(1.42,4.72)$ and $\left(\mathrm{Chi}^{2}=\right.$ 7.92 , df $\left.=4(p=0.09): I^{2}=49 \%\right)$, respectively. Moreover, the risk ratio at twelve weeks was (RR: $1.01(0.42,2.46)$, and heterogeneity was $\left(\mathrm{Chi}^{2}=8.55, \mathrm{df}=2(\mathrm{p}=0.01): \mathrm{I}^{2}=\right.$ $77 \%$ ). Overall, our findings indicate that EGF significantly promotes wound healing and it could be recommended as an effective and safe treatment for DFUs.
\end{abstract}

\section{Keywords}

Diabetic foot ulcer, Epidermal growth factor, Placebo, Metaanalysis

\section{Introduction}

Diabetes is a metabolic syndrome, which may be due to reduced insulin secretion, or defects in insulin function, or both. Due to pancreatic resistance, diabetes cells are unable to absorb glucose efficiently, resulting in hyperglycemia [1]. Following this, according to international diabetes federation 2017 [2], only diabetes is responsible for four million fatalities globally in 2017. As a results, according to published literature 382 million individuals were diagnosed with diabetes in 2013 [3], 415 million in 2015 [4], 425 million in 2017 [2], 463 million in 2019, and is projected to effect 578 million people in 2030 [5]. Consequently, from 2013 to 2030 , the average increase rate in diabetes patients is more than $52 \%$, this increasing tendency of morbidity and mortality is seen in patients with type 2 diabetes, which commonly leads to premature death.

The three main types of diabetes are type 1 diabetes (T1DM), type 2 diabetes mellitus (T2DM), and gestational diabetes mellitus (GDM). However, the onset of T2DM is more insidious accounting for roughly $90 \%$ of all cases [6]. Excessive thirst, hunger, weariness, sluggishness, weight loss in type one or progressive weight gain in type two, blurred vision, and passing more urine than normal are the most typical symptoms of diabetes. The most common acute consequences of diabetes are hyperglycemia, ketoacidosis, and diabetic

Citation: Rahim F, Yan X, Bibi N, Nawaz S, Ming Y (2022) Epidermal Growth Factor Outperforms Placebo in the Treatment of Diabetic Foot Ulcer: A Meta-Analysis. Int J Diabetes Clin Res 9:153. doi. org/10.23937/2377-3634/1410153

Accepted: February 16, 2022: Published: February 18, 2022

Copyright: (C) 2022 Rahim F, et al. This is an open-access article distributed under the terms of the Creative Commons Attribution License, which permits unrestricted use, distribution, and reproduction in any medium, provided the original author and source are credited. 
coma. The leading causes of diabetes are ageing, increased urbanization, genetics, and obesogenic profile [7]. People with a family history of diabetes, for example, have a $25 \%$ increased chance of inheriting type 2 diabetes from their parent [8]. Similarly, monozygotic twins are 90 percent more likely than heterozygotic twins to acquire T2DM later in life. Moreover, the incidence rates of T1D are also rising, contributing to the increase in diabetes prevalence worldwide [9]. However in depth knowledge for the specific cause of this rise is of paramount important.

Diabetes foot ulcers are expected to affect more than $19 \%$ of the world's adult population by 2030 , according to WHO projections [10]. DFUs are wounds in the dermis (the skin's deep blood vessels and collagen inner layer) that appear below the ankles of diabetics patients [11]. It is estimated that DFUs would affect 9.1 to 26.1 million diabetic patients each year across the globe [12]. Diabetic foot ulcers were found to be prevalent in $6.3 \%$ of the world's population, with North America (13\%) having the highest prevalence and Oceania having the lowest (3\%). In Asia, Europe, and Africa, the prevalence was 5.5\%, 5.1\%, and $7.2 \%$, respectively [13]. Indeed, diabetes mellitus is one of the most common causes of non-traumatic lower extremity amputation. Approximately $20 \%$ of diabetic foot infections that are mild to severe result in amputation [14]. DFU dramatically raised the risk of death in diabetic individuals by 2.5 times when compared to non-DFU patients [2,12]. Insufficient blood circulation due to malfunction of circulatory system significantly increase the incidence of diabetic foot ulcers. Therefore, set up an effective treatment strategy is imperative to treat DFUs more efficiently.

Several growth factors such as platelet-derived growth factor, fibroblast growth factor, epidermal growth factor (EGF), and peripheral blood mono nuclear cells and their combined applications have shown potentials in promoting ulcer healing [1517]. Inflammation, proliferation, and remodeling are the three stages of wound healing, each of which necessitates the coordination and integration of delicate and complicated biological activities. The growth factors participating in those biological events work by stimulating chemotaxis, cell proliferation, extracellular matrix deposition, angiogenesis, and tissue reconstruction. Several published literature have evaluated the curative effect of topical EGF and placebo on healing diabetic foot ulcers, but there are always contradictions in the evidence to distinguish the true therapeutic effect and safety issues of EGF and placebo in the treatment of DFUs $[18,19]$. Therefore, a comprehensive meta-analysis was conducted to evaluate the efficacy and safety of EGF and placebo on healing diabetic foot ulcers. The application of EGF, according to our hypothesis, outperforms placebo in facilitating the healing process of DFUs.

\section{Methodology}

The meta-analysis was reported according to the Preferred Reporting Items for Systematic Reviews and Meta-Analysis (PRISMA) statement [20].

\section{Literature search}

The relevant English literature search was performed in Cochrane Library, PubMed, EMBASE, and Google Scholar, from 2009 to 2021. Epidermal Growth Factor (EGF), Placebo, Diabetic Foot Ulcer, Diabetes, and Metaanalysis were among the terms used. Studies from the reference list were also incorporated in order to find more relevant material. Articles were located and checked on a variety of levels, including title, abstract, and full-text, final papers that met the inclusion criteria retrieved and included in the study, while those that did not were initially excluded.

\section{Eligibility criteria}

Inclusion criteria: Inclusion criteria for selecting studies include

1) Type 1 and 2 diabetic patients with foot wounds.

2) Literature mainly focused on complete healing time.

3) Any study design including double-blind, placebocontrolled trial, randomized control trails, retrospective study, prospective study.

4) Studies comparing epidermal growth factors and placebo in the treatment of diabetic foot ulcers.

\section{Exclusion criteria:}

1) Combine therapy.

2) Studies with no reported sample size.

3) Single study of epidermal growth factor or placebo treatment.

4) Non-human studies, reviews, protocols, and trials.

\section{Data extraction}

The following data were extracted from the eligible studies: initial author's name, year of publication, location, study time (start-to-end date), patients number, study design, patients characteristics (average age, sex, etc.), and treatment duration. Authors of articles with insufficient data were contacted for details. Those papers were deleted from the meta-analysis whose contacted author did not provide the required data. The full texts of potentially eligible studies were obtained and double-screened for eligibility by two potentials reviewers (Figure 1). Disagreements were resolved through discussion.

\section{Assessment of risk of bias}

To assess the quality of each study, the Cochrane 


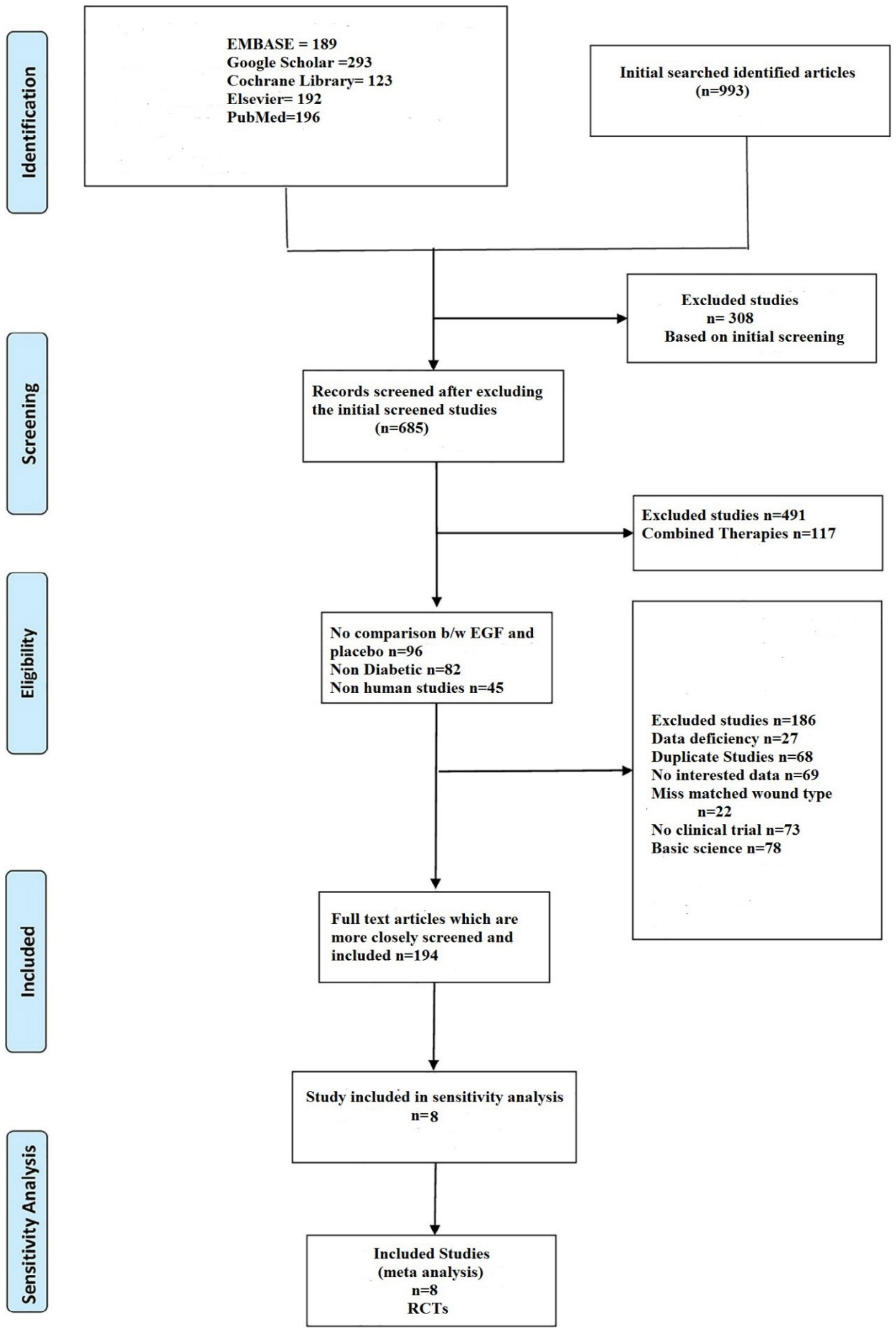

Figure 1: Summary of the included studies. 
risk, a biased evaluation tool in the Review Manager 5.4 programme for RCTs, and the Newcastle-Ottawa Scale (NOS) for cohort studies were employed. We assessed the random sequence generation (selection bias), allocation concealment (selection bias), blinding of participants and personnel (performance bias), blinding of outcome assessment (detection bias), incomplete outcome data (attrition bias), and other bias. Studies were categorized as having a high (red), unclear (yellow), or low (green) risk of bias in each domain. The risk of bias summary table and graph were regenerated by the RevMan software (version 5.4 Copenhagen: The Nordic Cochrane Center, the Cochrane Collaboration, 2014, Denmark). Besides, we drew a funnel plot to identify whether there was any publication bias.

\section{Statistical analyses}

Review Manager 5.4 were used to compile all of the data (RevMan, the Cochrane cooperation, Oxford, UK). For this meta-analysis, the heterogeneity between studies was assessed by Cochran (Q) and $I^{2}$ statistics, which expressed the percentage of variation between studies. $I^{2}$ was used to evaluate inter study heterogeneity. An $I^{2}$ value higher than $50 \%$ was considered to have statistically significant heterogeneity. We presented dichotomous outcomes as risk ratios (RRs) with their corresponding $95 \%$ confidence intervals (Cls). Fixed or random effect techniques are used in meta-analysis, depending on the degree of heterogeneity. The random effect approach is used to combine results when significant heterogeneity is identified in the data, whereas the fixed effect method is used when significant heterogeneity is not detected.

\section{Results}

\section{Study selection and characteristics}

In a systematic search of various electronic databases, a total of 993 articles were identified (EMBASE 189, Google Scholar 293, Cochrane Library 123, Elsevier 192, and PubMed 196). Following an initial screening, 308 studies were ruled out due to their titles. Following that, the remaining 685 articles were carefully screened, with 491 studies being excluded based on full text or abstract, including combination therapies ( $n=117$ ), no comparison between epidermal growth factor and placebo $(n=96)$, non-diabetic $(n=82)$, non-human studies $(n=45)$, basic science $(n=78)$, and no clinical trials $(n=73)$. The remaining 194 papers were evaluated more thoroughly, and 186 were eliminated due to data gaps $(n=27)$, duplicate studies $(n=68)$, content of studies without the desired outcome $(n=69)$, and wound type mismatch $(n=22)$. Finally, 8randomized controlled studies were included in the meta-analysis. Figure 1 shows the identified and retrieved articles in the study.

Eight randomized control trials (RCTs) that involved a total of 620 patients (337 in the EGF group) and 283 in the placebo group, were included. Except one study from Mexico and another from Cuba, the majority of the studies were from Asia. In these studies, patients received either EGF or placebo intervention, in addition to standard diabetic foot management. The EGF and placebo treatments were administrated by intralesional injection or topical application. All of the patients had type 1 or type 2 diabetes with DFUs. The patient's ages ranged from 20 to 75 -years-old. The majority of studies had a follow-up period of 4 to 14 weeks. Table 1 shows the basic characteristics of the included studies.

\section{Risk of bias and quality assessment}

Details on the risk of bias assessment are illustrated in Figure 2 and Figure 3. The quality assessment was performed on a total of eight studies included in qualitative analysis, with the results showing mostly a low and unclear risk of bias. By converting the Cochrane Risk of Bias Tool to Agency for Healthcare Research and Quality (AHQR) standard, our risk of bias assessment reported a fair quality. The funnel plot revealed a clear symmetric trend, indicating that publication bias was unlikely present (Figure 4).

\section{Four weeks healing rate of EGF and placebo}

Statistical analysis results are shown in Figure 5. Three publications including 85 patients were divided into two groups. The epidermal growth factor group recovered significantly faster than the placebo group after four weeks of treatment. For example, with 4-week treatment, the healing frequency in the placebo group was $17 \%$, whereas the healing frequency in the epidermal growth factor group was $34 \%$. Furthermore, there is a substantial difference in the healing rate between the epidermal growth factor and placebo groups after four weeks of treatment. Figure 5 shows a significant difference in risk ratio (RR: $3.04,[95 \% \mathrm{Cl}$ : $\left.0.50,18.44] \mathrm{I}^{2}=69 \%\right)$ and heterogeneity $\left(\mathrm{Chi}^{2}=6.46, \mathrm{df}\right.$ $\left.=\left.2(P=0.04)\right|^{2}=69 \%\right)$.

\section{Eight weeks healing rate of EGF and placebo}

The healing rate of epidermal growth factor and the placebo group is compared in five articles. There were 123 patients in the epidermal growth factor group and 125 in the placebo group. After eight weeks of therapy, the percentage of patients who healed was $79 \%$ in the epidermal growth factor group while $25 \%$ in the placebo group, indicating a significant difference (Figure 6). The proportion of complete ulcer healing with EGF was significantly higher than that of placebo (RR: $2.59,[95 \%$ $\left.\mathrm{Cl}: 1.42,4.72] \mathrm{I}^{2}=49 \%\right)$ and $\left(\mathrm{Chi}^{2}=7.92, \mathrm{df}=4(p=0.09)\right.$ : $\left.\mathrm{I}^{2}=49 \%\right)$ (Figure 6$)$.

\section{Twelve weeks healing rate of EGF and placebo}

A total of three papers compare the healing rate of EGF and placebo groups after twelve weeks of therapy. 


\begin{tabular}{|c|c|c|c|c|c|c|c|c|}
\hline 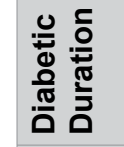 & 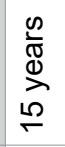 & $\mathbb{z}$ & $\mathbb{z}$ & $\mathbb{z}$ & $\S$ & $\Sigma$ & $\mathbb{z}$ & $\begin{array}{ll}m \\
\stackrel{0}{a} \\
\stackrel{0}{\alpha}\end{array}$ \\
\hline
\end{tabular}

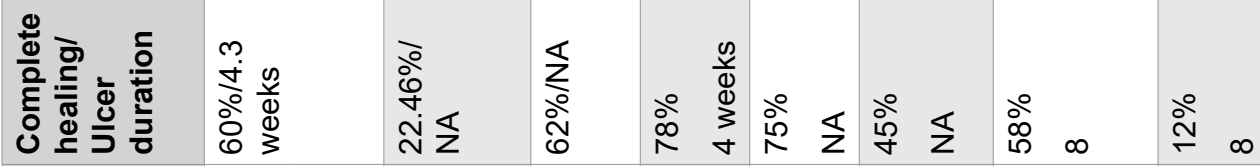

\begin{tabular}{|c|c|c|c|c|c|c|c|c|}
\hline 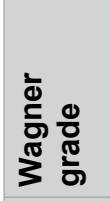 & $\begin{array}{ll} & \\
0 \\
\infty \\
\infty \\
0 \\
0 \\
0 \\
0 \\
0 \\
0\end{array}$ & $\begin{array}{l}N \\
\vdots \\
\frac{1}{0} \\
\frac{0}{8} \\
\frac{\pi}{0} \\
0\end{array}$ & $\begin{array}{l}\bar{\delta} \\
\overline{0} \\
\overline{0} \\
0 \\
0\end{array}$ & 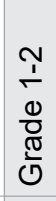 & $\begin{array}{l}N \\
0 \\
\frac{0}{0} \\
\frac{\pi}{0} \\
0\end{array}$ & 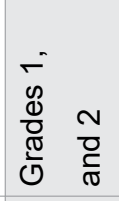 & $\frac{\mathbb{s}}{z}$ & 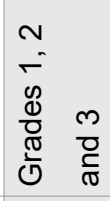 \\
\hline
\end{tabular}

\begin{tabular}{|c|c|c|c|c|c|c|c|c|c|}
\hline 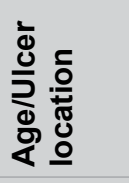 & \multicolumn{2}{|l|}{ 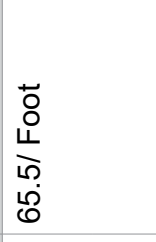 } & 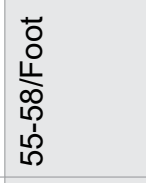 & 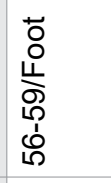 & 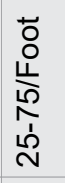 & $\begin{array}{l}\overrightarrow{0} \\
\text { بै } \\
\text { ⿳亠丷厂 } \\
\text { ì }\end{array}$ & 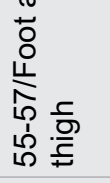 & 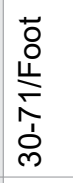 & $\begin{array}{l}\overrightarrow{0} \\
\stackrel{0}{\omega} \\
\stackrel{1}{0} \\
\infty \\
\omega\end{array}$ \\
\hline 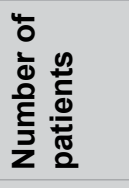 & 导 & & ८) & $\hat{\theta}$ & is & 8 & i̊ & 8 & ले \\
\hline 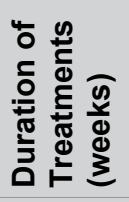 & $\begin{array}{l}\frac{\mathscr{D}}{\mathbb{D}} \\
\frac{\Phi}{\omega} \\
\sum_{\infty}\end{array}$ & & 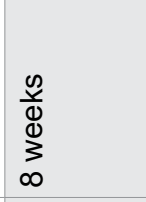 & 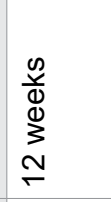 & 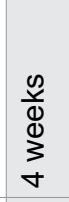 & 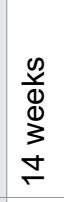 & 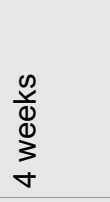 & 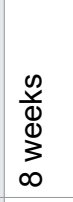 & 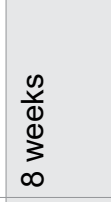 \\
\hline 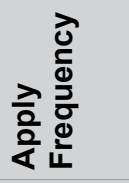 & 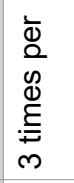 & & 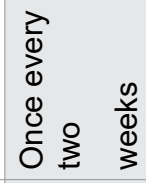 & 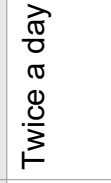 & $\mathbb{z}$ & $\mathbb{z}$ & $\mathbb{z}$ & $\mathbb{z}$ & 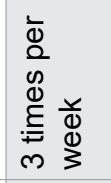 \\
\hline 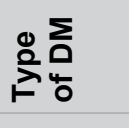 & 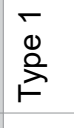 & & 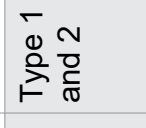 & 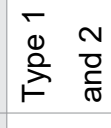 & $\mathbb{s}$ & $\mathbb{z}$ & 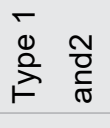 & $\mathbb{z}$ & 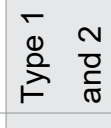 \\
\hline 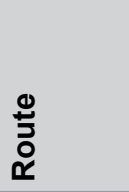 & 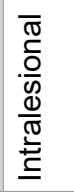 & 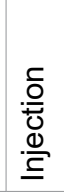 & 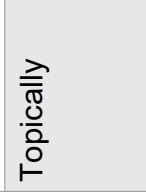 & 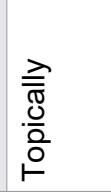 & 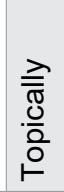 & 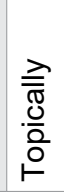 & 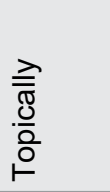 & 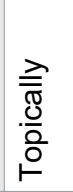 & 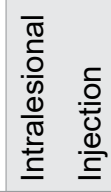 \\
\hline
\end{tabular}

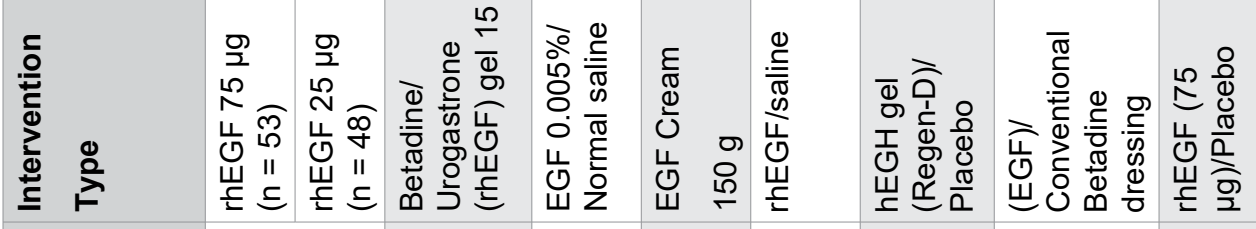

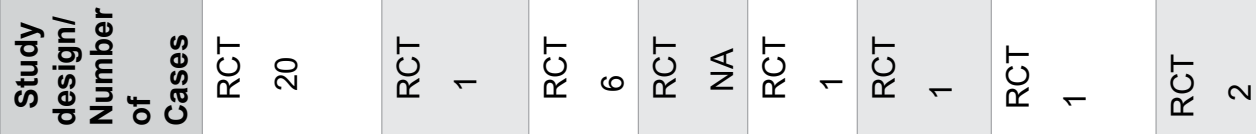

\begin{tabular}{|c|c|c|c|c|c|}
\hline 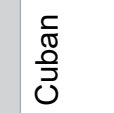 & $\frac{\underline{\underline{z}}}{\underline{\underline{\underline{z}}}}$ & 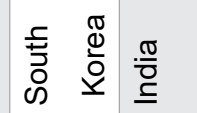 & $\frac{\underline{\underline{\alpha}}}{\underline{\underline{\underline{z}}}}$ & $\frac{\tilde{z}}{\underline{\underline{\underline{z}}}}$ & 离 \\
\hline 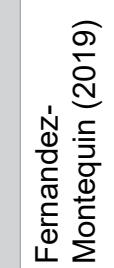 & 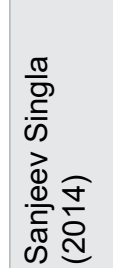 & 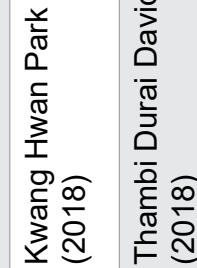 & 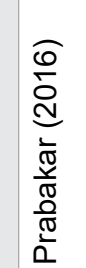 & 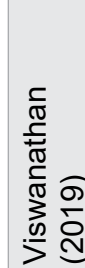 & 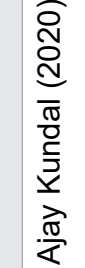 \\
\hline
\end{tabular}




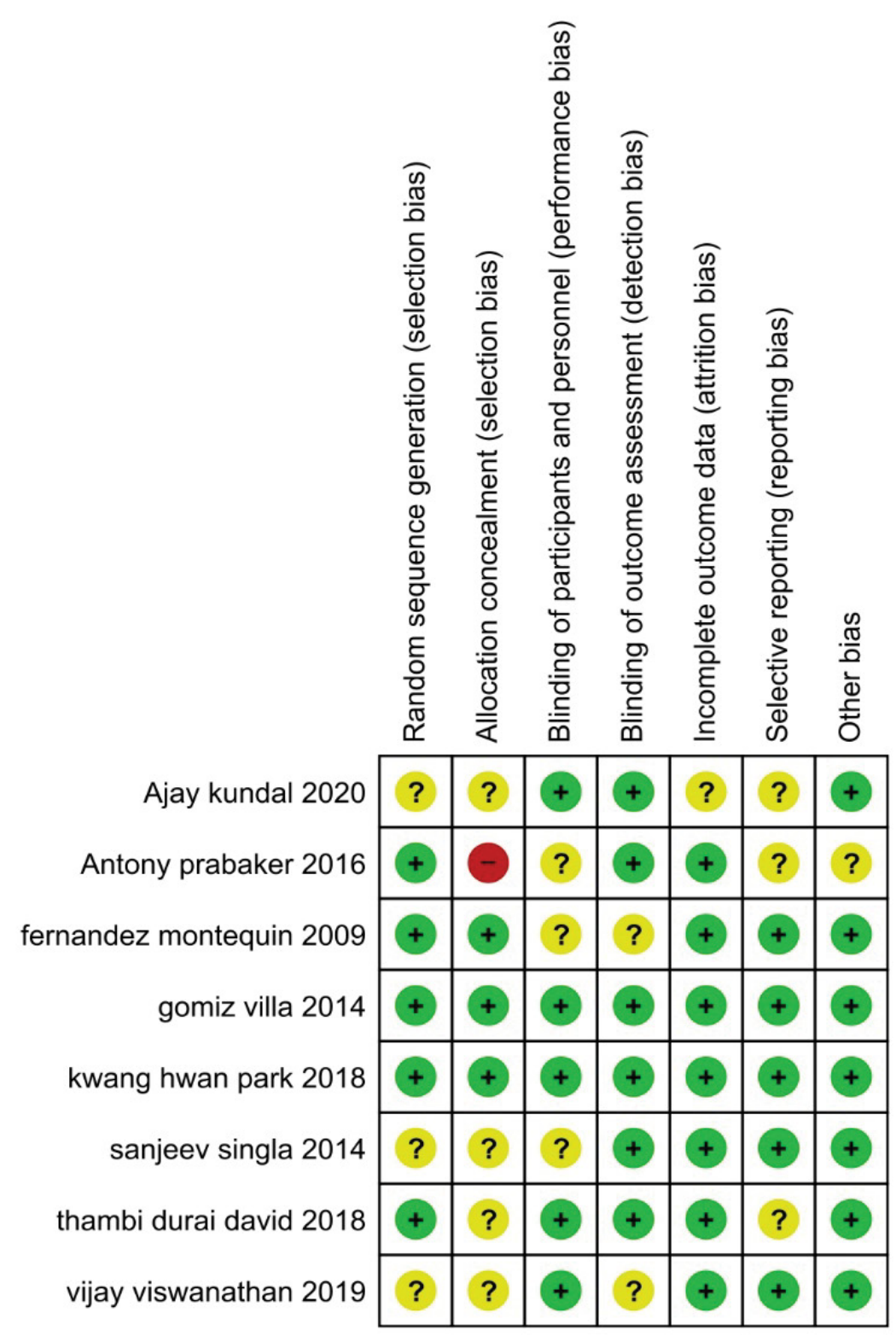

Figure 2: The summary of the risk of bias for each study that was included.

Random sequence generation (selection bias)

Allocation concealment (selection bias)

Blinding of participants and personnel (performance bias)

Blinding of outcome assessment (detection bias)

Incomplete outcome data (attrition bias)

Selective reporting (reporting bias)

Other bias
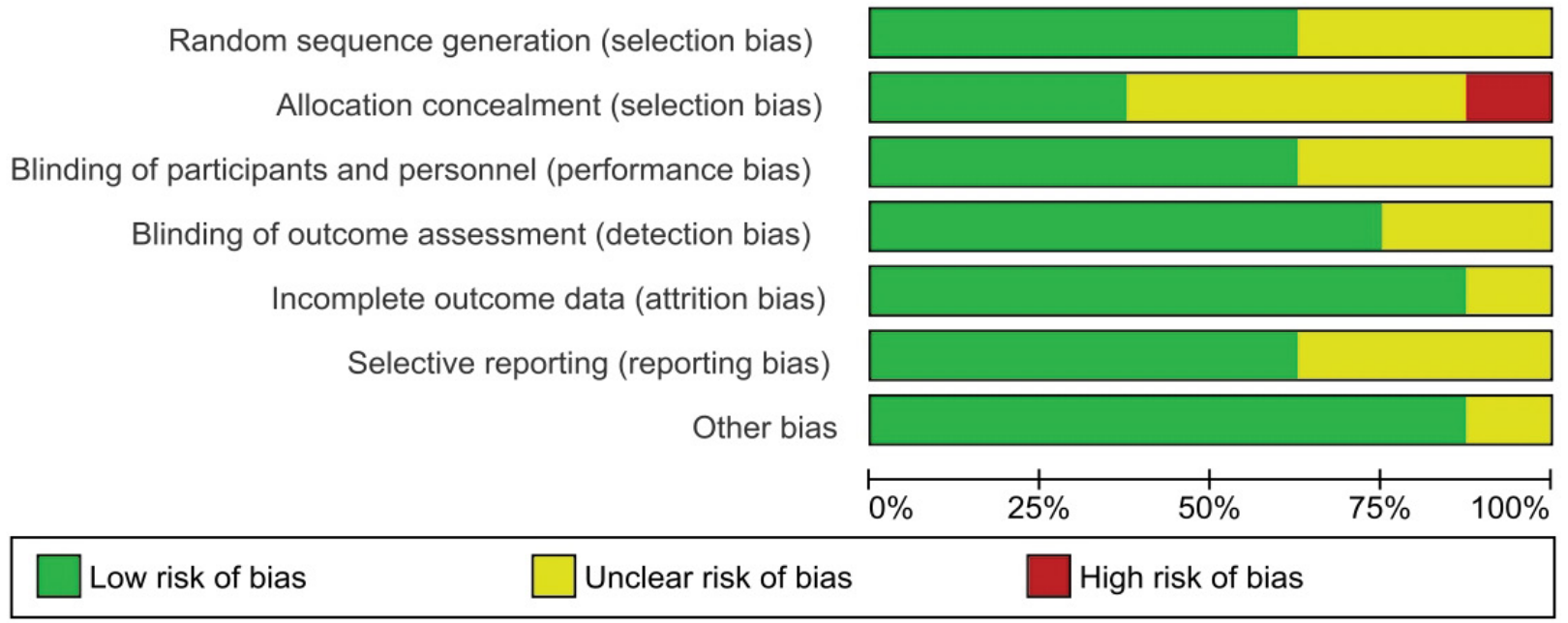

Figure 3: The risk of bias graph was calculated for each study. 


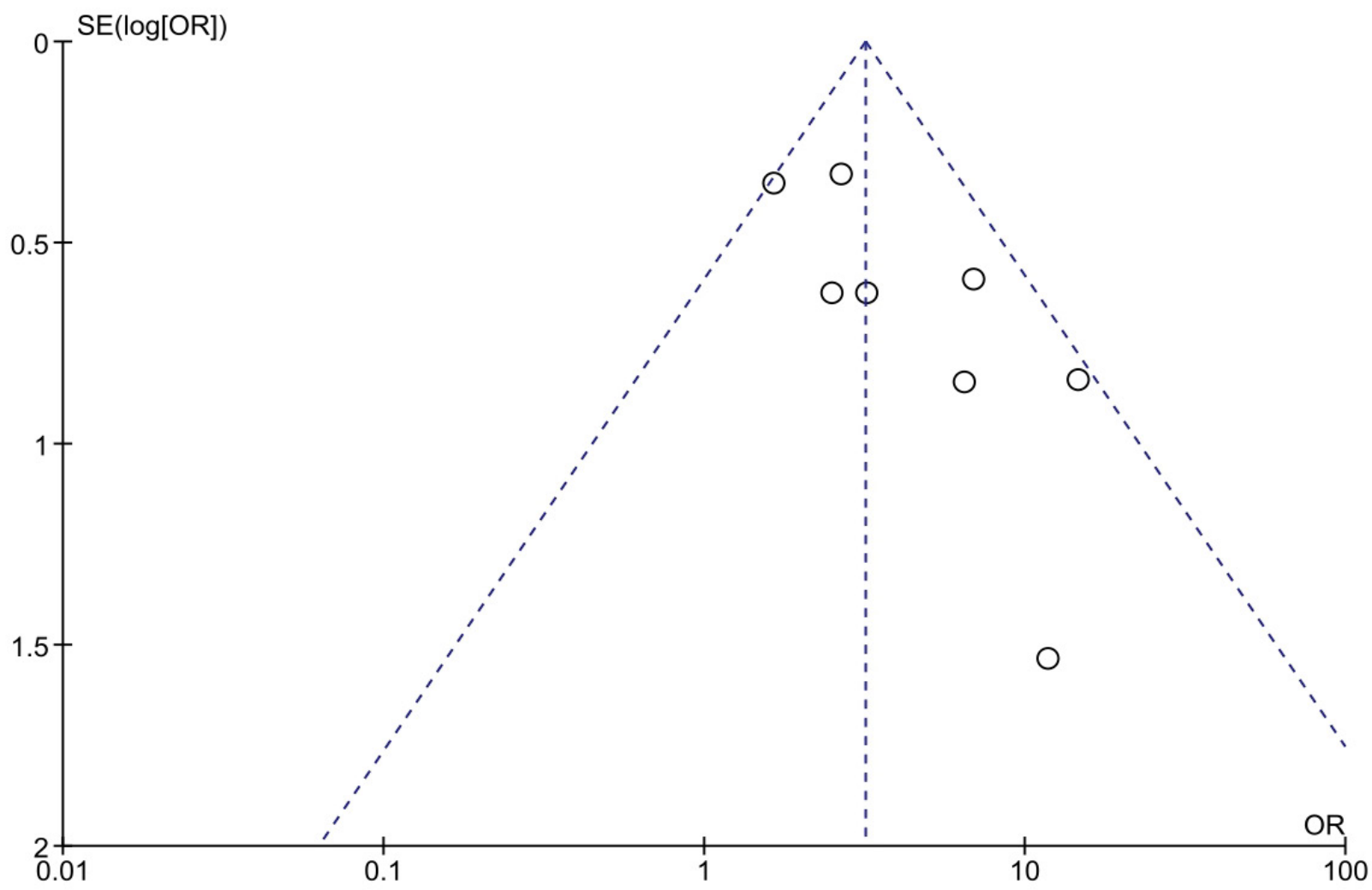

Figure 4: Funnel plot.

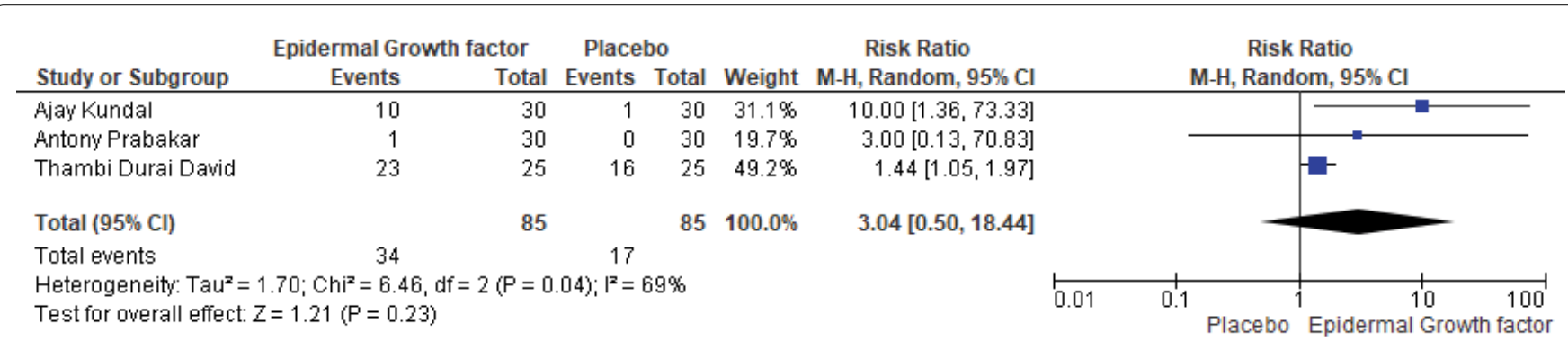

Figure 5: Four-week healing rates of EGF and placebo.

Abbreviations: Cl: Confidence Interval; df: Degrees of Freedom; EGF: Epidermal Growth Factor

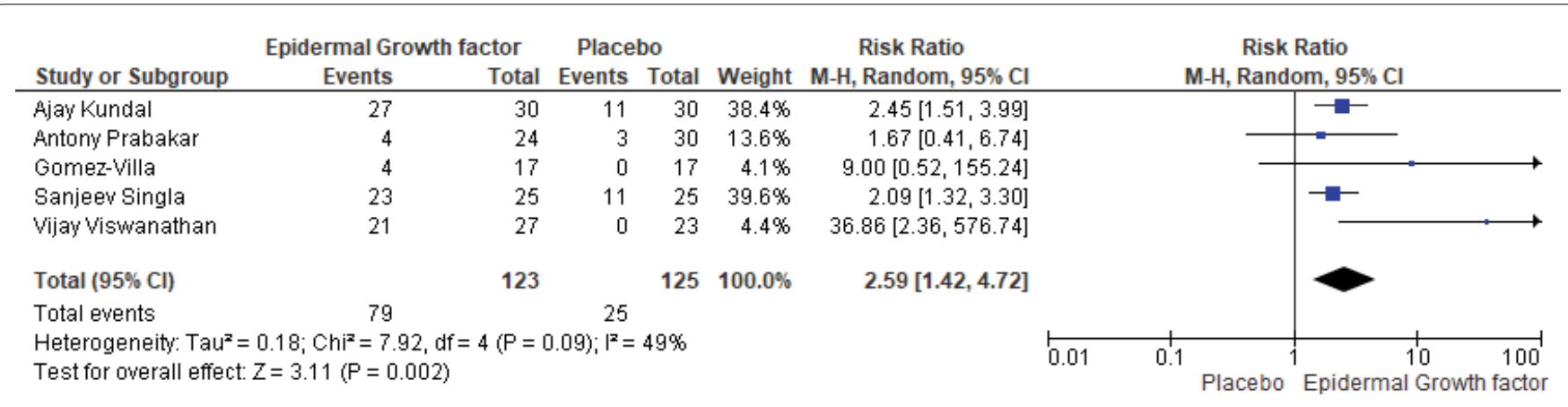

Figure 6: Eight weeks healing rate of EGF and placebo.

The EGF group received 134 patients, while the placebo group received 135 . There was a clear difference between the epidermal growth factor and placebo groups after twelve weeks of treatment (Figure 7). The epidermal growth factor group had a healing rate of $71 \%$, while the placebo group had a healing rate of
$58 \%$. Furthermore, a significant difference in risk ratio and heterogeneity were noted, with risk ratio (RR: 1.01, [95\% Cl: $\left.0.42,2.46] \mathrm{I}^{2}=77 \%\right)$ and heterogeneity (Chi ${ }^{2}=$ $\left.8.55, \mathrm{df}=2(p=0.01): \mathrm{I}^{2}=77 \%\right)$.

Complete healing rate with EGF versus placebo 


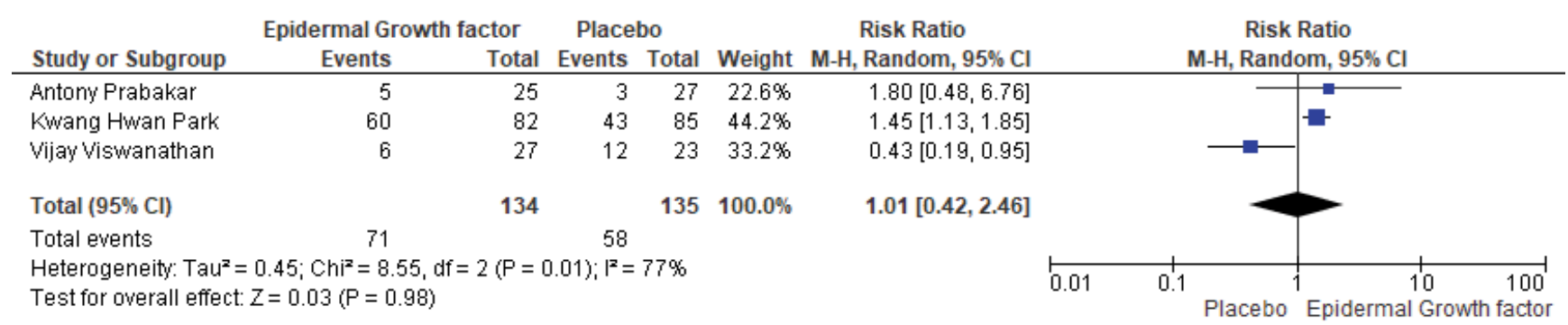

Figure 7: Twelve-week healing rate of EGF and placebo.

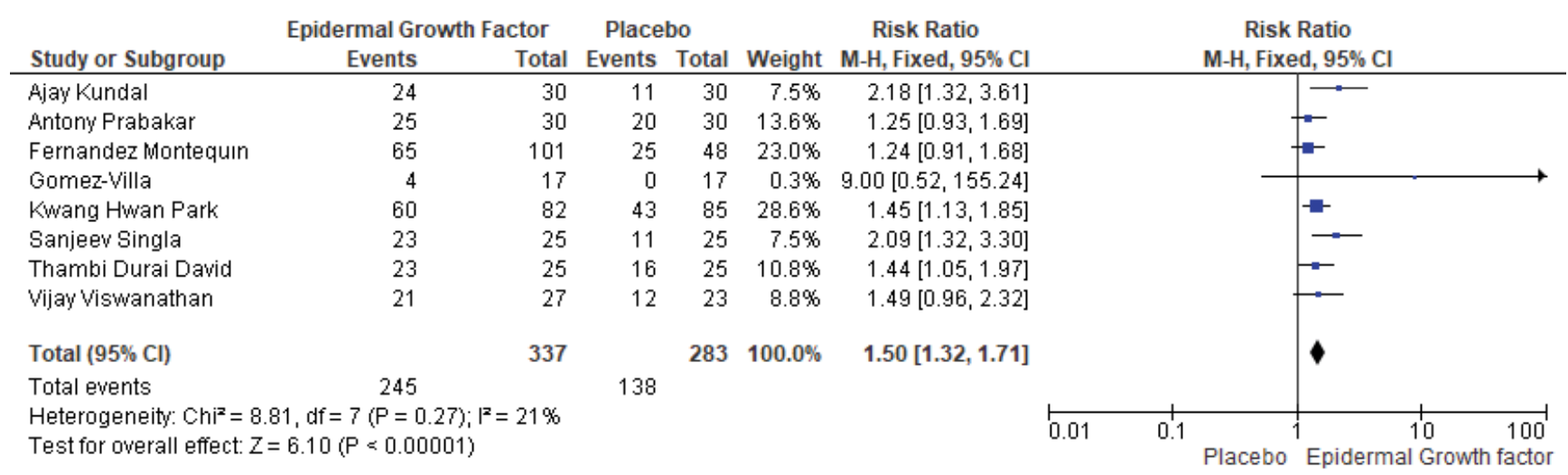

Figure 8: Complete healing rate of EGF and placebo in diabetic foot ulcer patients.

Statistical analysis results for complete healing rate of diabetic foot ulcers in patients treated with EGF versus placebo is shown in Figure 8. The complete healing rate study includes a total of 8 publications, with 620 patients demonstrating a complete healing rate, 337 of them were in the EGF group and 283 in the placebo group. For instance in the epidermal growth factor group a total of 245 (out of 337) events occurs, while in placebo group a total of 138 (283) events occurs. The risk ratio and heterogeneity were (RR: 1.50, [95\% Cl: $\left.1.32,1.71] \mathrm{I}^{2}=21 \%\right)$ and heterogeneity $\left(\mathrm{Chi}^{2}=8.81, \mathrm{df}=\right.$ $\left.7(p=0.27): 1^{2}=21 \%\right)$. Overall, the complete healing rate of epidermal growth factor was significantly higher than that of placebo and it could be used as a recommended first line therapy for treatment of DFUs (Figure 8).

\section{Discussion}

This study was carried out to clarify the efficacy and safety of EGF and placebo in the treatment of diabetic foot ulcer. A total of 620 diabetic patients with foot ulcers (337 randomized to the EGF group and 283 to the placebo group) were included in the meta-analysis. On the other hand, several meta-analyses have been published in the last decade [21-23]. However, previous published studies, frequently share some common limitations. For example, the quality of risk-of-bias was insufficient because of the unknown performance of bias since the patients were not blinded, which could lead to an overestimation of the study's quality. Another disadvantage is that most studies do not contain any relevant literature at all. Furthermore, past researches have employed protocol analysis [22], which has the potential to disrupt the baseline character balance and overestimate treatment outcomes. Therefore, taken all of the inherent limitations into account, a meta-analysis was undertaken based on eight trials that included data from various nations aiming to compare EGF vs. placebo alone. The EGF group consisted of 337 patients, while the placebo group consisted of 283 people. Our results indicated that the use EGF significantly improves the healing rate compared with placebo in the treatments of DFUs. For instance, the EGF group shows a complete healing rate of $71 \%$ while the placebo group shows a complete healing rate of $58.7 \%$. Our findings are similar to those of Quoc Van Phu Bui, et al. [22], who discovered that epidermal growth factor therapy is superior over placebo. Furthermore, Viswanathan, et al. [24], discovered a substantial difference between epidermal growth factors and placebo therapy. For example, the EGF group had a complete healing rate of $78 \%$, whilst the group of placebo had a complete healing rate of $52 \%$. The most positive conclusion in our study is that EGF improves foot ulcer healing considerably. As a result, as compared to placebo, EGF therapy results in a faster recovery of the wound. The significance of growth factors in wound repair could be one explanation for the remarkable therapeutic impact. EGF promotes epidermal cell proliferation by stimulating glycolysis, mitosis, and protein synthesis [25]. By causing inflammatory cells to relocate to ulcer sites, EGF can enhance the wound microenvironment and tissue nutrition. Furthermore, Thambi Durai David, 
et al. [26] noted that patients treated with EGF had a substantially higher rate of complete healing rates than those treated with placebo i.e. the percentage of total recovery in the EGF and placebo groups was $71.2 \%$ and $48.9 \%$, respectively (Figure 8 ). Over the course of four weeks, a significant difference was found in cure rates between the EGF and placebo groups, ranging from $3-92 \%$ to $0-65 \%$, respectively. Our obtained results are compatible with the findings of the previous metaanalysis [21]. For example, Yang, et al. [21], found that, EGF therapy outperformed placebo and the healing rate was $23 \%$ in the EGF group and $10 \%$ in the placebo group (Figure 6). Consequently, after eight weeks of therapy, there was a substantial difference between the EGF and placebo groups, with healing rates of $16-92 \%$ and $0-25 \%$, respectively. Following this in a recent meta-analysis, Kumar, et al. [27], found a significant difference between the EGF group and the placebo group, i.e. in the EGF group, complete wound healing was $76 \%$, compared to $20 \%$ in the placebo group. Moreover, the average recovery time for totally healed ulcers was eleven weeks (Figure 7). However, wound healing in the EGF and placebo groups differed significantly after 12 weeks of therapy. The EGF group had a healing rate of $20-73 \%$, while the placebo group had a healing rate of $11-52 \%$. According to previous research by Yang, et al. [28], EGF is far superior to the placebo group, with a complete cure rate of $23.5-95.3 \%$ in EGF and 0 to $52.1 \%$ in the placebo group. Overall, our findings provide meaningful information to current treatment scenarios of DFUs. As a result, based on current evidence, EGF administration to DFUs is considered as effective and safe.

The most important strengths of our meta-analysis is that; first to our knowledge with eight RCTs this is the most comprehensive meta-analysis on comparing EGF and placebo for DFUs. We also looked for suitable references and emailed the authors for the missing information. Our meta-analysis includes several current clinical studies, which provided more evidence than the prior meta-analysis. This is significant because the number of trials comparing EGF to placebo in the treatment of DFU is currently limited. However, the possible limitations of this research are that, even though publication bias analysis was performed, there are still some potential biases. Some of the literatures included were of poor quality. Although the authors reported that their studies were randomized, the random sequences and blind details were not described in the original articles. The origin of the works did not correspond to a homogeneous recruitment. The economic analysis is important for patients with long diabetic history, especially in developing countries. However, most of the trials didn't provided cost-effectiveness data. The amount of data on adverse effects was also limited, and hence we could not elicit the most common side effect experienced as a result of EGF and placebo treatment.

\section{Conclusions}

Compared to placebo therapy, EGF significantly accelerate the healing of diabetic foot ulcers at 4-12 weeks of treatment. The EGF has the potential to improve ulcer rehabilitation and speed wound healing. This conclusion, however, should be approached with caution. More well-designed clinical trials in different populations with long follow-up time are required to further examine the topical EGF therapy in management of diabetic foot ulcer in the future.

\section{Conflict of Interest}

The authors declare no potential conflicts of interest.

\section{Funding}

This work is supported by key Research \& development projects of Ningxia Autonomous region, 2019, Grant number: 2019BEG03069.

\section{References}

1. Unuofin JO, Lebelo SL (2020) Antioxidant effects and mechanisms of medicinal plants and their bioactive compounds for the prevention and treatment of type 2 diabetes: An updated review. Oxid Med Cell Longev 2020: 1356893.

2. International Diabetes Federation (2017) IDF Diabetes Atlas. ( $\left.8^{\text {th }} \mathrm{edn}\right)$, International Diabetes Federation, Brussels, Belgium.

3. International Diabetes Federation (2013) IDF Diabetes Atlas. $\left(6^{\text {th }} \mathrm{edn}\right)$, International Diabetes Federation, Brussels, Belgium.

4. International Diabetes Federation (2015) IDF Diabetes Atlas. ( $7^{\text {th }}$ edn), International Diabetes Federation, Brussels, Belgium.

5. Saeedi P, Petersohn I, Salpea P, Malanda B, Karuranga $S$, et al. (2019) Global and regional diabetes prevalence estimates for 2019 and projections for 2030 and 2045: Results from the International Diabetes Federation Diabetes Atlas, 9th edition. Diabetes Res Clin Pract 157: 107843.

6. Davis IC, Ahmadizadeh I, Randell J, Younk L, Davis SN (2017) Understanding the impact of hypoglycemia on the cardiovascular system. Expert Rev Endocrinol Metab 12: 21-33.

7. Alotaibi A, Perry L, Gholizadeh L, Al-Ganmi A (2017) Incidence and prevalence rates of diabetes mellitus in Saudi Arabia: An overview. J Epidemiol Glob Health 7: 211-218.

8. Klein BE, Klein R, Moss SE, Cruickshanks KJ (1996) Parental history of diabetes in a population-based study. Diabetes Care8: 827-830.

9. Patterson CC, Dahlquist GG, Gyurus E, Green A, Solte'sz G (2009) EURODIAB Study Group. Incidence trends for childhood type 1 diabetes in Europe during 1989-2003 and predicted new cases 2005-20: A multicentre prospective registration study. Lancet 9680: 2027-2033.

10. Grennan D (2019) Diabetic foot ulcers. JAMA 321: 114.

11. Armstrong DG, Boulton AJM, Bus SA (2017) Diabetic foot ulcers and their recurrence. N Engl J Med 376: 2367-2375.

12. Walsh JW, Hoffstad OJ, Sullivan MO, Margolis DJ (2016) Association of diabetic foot ulcer and death in a population- 
based cohort from the United Kingdom. Diabet Med 33: 1493-1498.

13. Zhang P, Lu J, Jing Y, Tang S, Zhu D, et al. (2017) Global epidemiology of diabetic foot ulceration: A review and metaanalysis. Ann Med 49: 106-116.

14. International Diabetes Federation (2012) The global burden. IDF diabetes atlas. ( $5^{\text {th }}$ edn).

15. Onodera R, Teramukai S, Tanaka S, Kojima S, Horie T, et al. (2011) Bone marrow mononuclear cells versus G-CSFmobilized peripheral blood mononuclear cells for treatment of lower limb ASO: Pooled analysis for long-term prognosis. Bone Marrow Transplant 46: 278-284.

16. Choi SM, Ryu HA, Lee KM, Kim HJ, Park IK, et al. (2016) Development of stabilized growth factor-loaded hyaluronatee collagen dressing (HCD) matrix for impaired wound healing. Biomater Res 20: 9.

17. Lee CH, Liu KS, Chang SH, Chen WJ, Hung KC, et al. (2015) Promoting diabetic wound therapy using biodegradable rhPDGF-Loaded nanofibrous membranes: CONSORTcompliant article. Medicine 94: e1873.

18. Hong JP, Jung HD, Kim YW (2006) Recombinant human epidermal growth factor (EGF) to enhance healing for diabetic foot ulcers. Ann Plast Surg 56: 394-398.

19. Persiani F, Paolini A, Camilli D, Mascellari L, Platone A, et al. (2018) Peripheral blood mononuclear cells therapy for treatment of lower limb ischemia in diabetic patients: A single-center experience. Ann Vasc Surg 53: 190-196.

20. Liberati A, Altman DG, Tetzlaff J, Mulrow C, Gøtzsche PC, et al. (2009) The PRISMA statement for reporting systematic reviews and meta-analyses of studies that evaluate healthcare interventions: Explanation and elaboration. BMJ 339: b2700.
21. Yang S, Geng Z, Ma K, Sun X, Fu X (2016) Efficacy of topical recombinant human epidermal growth factor for treatment of diabetic foot ulcer: A systematic review and meta-analysis. Int J Low Extrem Wounds 15: 120-125.

22. Thien Quoc B, Quoc VPB, Nemeth D, Hegyi P, Szakács $Z$, et al. (2019) Epidermal growth factor is effective in the treatment of diabetic foot ulcers: Meta-analysis and systematic review. Int J Environ Res Public Health 16: 2584.

23. Yang Q, Zhang Y, Yin H, Lu Y (2020) Topical recombinant human epidermal growth factor for diabetic foot ulcers: A meta analysis of randomized controlled clinical trials. Ann Vasc Surg 62: 442-451.

24. Viswanathan V, Udyama J, Babu M (2019) Efficacy of recombinant human epidermal growth factor (Regen-D 150) in healing diabetic foot ulcers: A hospital-based randomized controlled trial. Int J Low Extrem Wounds 19: 158-164.

25. Mohan VK (2007) Recombinant human epidermal growth factor (REGEN-D 150): Effect on healing of diabetic foot ulcers. Diabetes Res Clin Pract 78: 405-411.

26. Thambi DD, Jayalal JA, Selwyn JK (2019) The role of epidermal growth factor cream in healing of diabetic foot ulcer- comparative analytical study in South India. International Journal of Health Sciences \& Research, 8.

27. Kumar A, Sinha S, Khandelwal B (2020) Efficacy of topical recombinant epidermal growth factor as compared to povidone iodine on chronic diabetic foot ulcers. International Journal of Health and Clinical Research 3: 82-88.

28. Yang Q, Zhang Y, Yin H, Lu Y (2019) Topical recombinant human epidermal growth factor for diabetic foot ulcers: $A$ meta-analysis of randomized controlled clinical trials. Ann Vasc Surg 62: 442-451. 